\title{
Identifying the absolute orientation of a low-symmetry surface in real space
}

\author{
Stefan Kuhn, ${ }^{1}$ Markus Kittelmann, ${ }^{1}$ Yoshiaki Sugimoto, ${ }^{2}$ Masayuki Abe,${ }^{3}$ Angelika Kühnle, ${ }^{1}$ and Philipp Rahe,${ }^{4}$ \\ ${ }^{1}$ Johannes Gutenberg-Universität Mainz, Institut für Physikalische Chemie, Duesbergweg 10-14, 55099 Mainz, Germany \\ ${ }^{2}$ Graduate School of Engineering, Osaka University, 2-1 Yamada-Oka, Suita, Osaka 565-0871, Japan \\ ${ }^{3}$ Graduate School of Engineering Science, Osaka University, 1-3 Machikaneyama, Toyonaka, Osaka 560-8531, Japan \\ ${ }^{4}$ Department of Physics and Astronomy, The University of Utah, Salt Lake City, Utah 84112-0830, USA \\ (Received 22 July 2013; revised manuscript received 22 September 2014; published 5 November 2014)
}

\begin{abstract}
We present atomic-scale force interaction data acquired on an insulating low-symmetry substrate, namely the calcite(10.4) surface. A pronounced asymmetry of the imaged species is apparent along the vertical axis, which will be identified as a tilted band of purely attractive short-range interaction forces. An independent determination of the absolute surface orientation by an optical method suggests that the observed asymmetry is related to the tilt of the carbonate groups within the surface, which, in turn, is linked to the surface orientation. Thus our noncontact atomic force microscopy based work presents the determination of the absolute orientation for a low-symmetry surface.
\end{abstract}

DOI: 10.1103/PhysRevB.90.195405

PACS number(s): 68.35.B-, 68.37.Ps

\section{INTRODUCTION}

Investigating the properties of metallic or insulating surfaces at the atomic scale has progressed enormously during the last decades. Especially with the advent of scanning probe microscopy (SPM) techniques [1,2], studies in real space of bare surface terminations [3-5] or molecular superstructures [6,7] including the study of single molecules $[8,9]$ became possible. Among the long list of relevant surface properties, surface symmetry is probably the most decisive parameter. A knowledge of this basic surface property is of utmost importance for understanding key aspects of physicochemical processes, especially for materials relevant in material science or abound in the natural environment. In this context, a particularly interesting crystal is calcite due to its abundance in remarkable organic/inorganic hybrid materials known as biominerals.

Interestingly, most surfaces studied with noncontact atomic force microscopy (NC-AFM) so far contain a large number of surface symmetry elements and the absolute surface orientation is commonly directly identified from imaging the unit cell with SPM. To name two examples, the prototypical dielectric substrates $\mathrm{KBr}(001)$ and $\mathrm{NaCl}(001)$, which have routinely been used for atomic-scale investigations [10-13], molecular adsorption studies [6] or surface patterning [14], belong to the point group Fm3m [15]. In contrast, the absolute surface orientation can firsthand remain undisclosed in images from surfaces exhibiting a low symmetry. Especially in the NC-AFM images presented so far from the calcite(10.4) and the related dolomite(10.4) surface [16,17], one surface direction remains undetermined.

Here, we unravel this key symmetry property of the calcite(10.4) surface from short-range force data acquired with NC-AFM. The data reveal the orientation of the symmetry breaking, tilted carbonate ion $\mathrm{CO}_{3}^{2-}$ in the calcite(10.4) surface. A macroscopic optical method allows to confirm this orientation by independent means.

\footnotetext{
${ }^{*}$ Current address: School of Physics and Astronomy, The University of Nottingham, Nottingham NG7 2RD, UK; philipp.rahe@nottingham.ac.uk
}

We will first describe in Sec. II the complex data acquisition and analysis methodology. Section III introduces the calcite(10.4) surface with a special focus on the symmetry properties. The macroscopic approach to determine the absolute orientation is outlined in Sec. IV and followed by the microscopic observations, namely the tip-sample force interactions, including a model for the underlying imaging mechanism (Secs. V and VI).

\section{METHODS}

Data were acquired under ultrahigh vacuum conditions at room temperature using a VT AFM 25 (Omicron Nanotechnology, Taunusstein, Germany) atomic force microscope in beam-deflection configuration [18] operated in the frequencymodulated [19] noncontact mode. We used $\mathrm{Ar}^{+}$sputtered Si probes from Nanoworld (Neuchâtel, Switzerland) with frequencies around $300 \mathrm{kHz}$, excited to an amplitude of about $16.9 \mathrm{~nm}$. Experiments were performed in the so-called force mapping mode, where the data were systematically acquired along the vertical $(Z)$ and horizontal $(X$ or $Y)$ axes, resulting in high-resolution two-dimensional slices $(Z X$ or $Z Y$ mode) [20].

Utmost measurement stability is required and established at room temperature using a home-built atom-tracking system [21] for drift measurement and compensation [22]. A complex scan protocol of alternating steps of drift compensation and data acquisition is implemented [21] and described in Sec. II A.

The total tip-sample interaction force $F_{z}$ and potential $U_{\text {total }}$ are recovered from the frequency shift $\Delta f$ data using the Sader-Jarvis formulas [23]. We additionally calculate the lateral forces from $U_{\text {total }}$ and extract [24] the short-range interaction forces $F_{z, \mathrm{SR}}$ from a careful subtraction of the van der Waals contribution. The employed methodology is outlined in Secs. II B and II C.

\section{A. 2D force mapping scan protocol}

We employ the so-called curve-by-curve force mapping method, where single $\Delta f(z)$ curves are recorded on a lateral 
(a) (b)
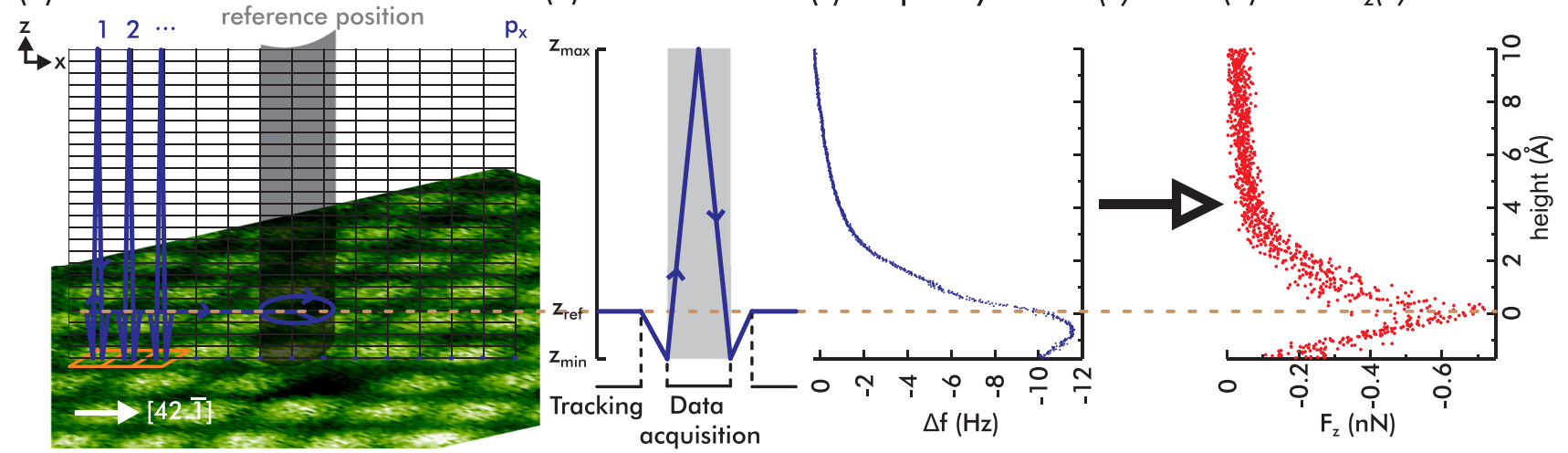

FIG. 1. (Color online) (a) Scan protocol for the curve-by-curve acquisition mode, where alternating steps of tracking and data acquisition are used. The total number of $\Delta f(z)$ curves is $p_{x}$ for a single slice. The dashed orange line marks the reference tip-sample distance $z_{\text {ref }}$ given by the $\Delta f$ setpoint. (b) Timing and $Z$ trajectory of the data acquisition. (c) Exemplary $\Delta f(z)$ curve, showing the frequency shift $\Delta f$ as a function of tip-sample distance $z$. (d) $F_{z}(z)$ curve extracted from the raw $\Delta f(z)$ data in (c) with applied filters (see main text).

grid (along the $X$ and/or $Y$ axes). In this mode, the $Z$ direction is the fast scan direction along which the data are sampled. We favor the curve-by-curve method over other strategies such as a layer-by-layer approach [25] for three reasons. First, tip changes are easily identified while acquiring the data. Second, if a tip change occurs within the data acquisition, it is possible to use the data up to the point of the tip change for the force recovery. Third, we expect to reduce artifacts caused by creep and hysteresis of the scanning piezos as well as residual drift in the curve-by-curve approach. Especially, we sample the most sensitive data nearby the $\Delta f(z)$ and $F_{z}(z)$ curve minima without vastly repositioning the tip before data acquisition.

Severe drift conditions usually present at room temperature render a precise and effective drift compensation strategy indispensable when acquiring dense and high-resolution force interaction data. We employ the atom-tracking principle [26,27] in a home-built implementation [21] for the drift measurement. This technique pins the scanning tip to a specific sample site by a lock-in detection with subangstrom precision. Based on the resulting drift velocities from following a surface site, we compensate for the unwanted movement by a feedforward routine [22]. Before acquiring the actual $2 \mathrm{D}$ slice ( $Z X$ mode), we scanned several images in regular AFM imaging ( $X Y$ mode) and repeatedly corrected for thermal drift. This procedure not only ensures to reduce the residual drift already to a minimum, but especially decreases scanner creep. The residual drift due to the nonlinear contribution was typically significantly below $100 \mathrm{pm} / \mathrm{min}$ before starting the $Z X$ data acquisition.

Figures 1(a) and 1(b) visualize the scan protocol itself. We use the atom-tracking technique to center the tip at a reference position usually located in the middle of the slice. In this case, the reference position is assigned to a single atomic surface site and the tip is moved to every lateral position from this site to acquire a single $\Delta f(z)$ curve. The data acquisition requires $t_{\text {acq }}$ per curve. After moving the tip back to the reference position, the atom tracking compensates for nonlinear drift and creep contributions by precisely re-centering the tip on the selected feature for time $t_{\text {track }}$. In the herein presented data, residual and nonlinear drift contributions were in the order of approximately $4 \mathrm{pm} / \mathrm{min}$ along $X$ and approximately 7 $\mathrm{pm} / \mathrm{min}$ along $Z$ and cause an error in the absolute lateral and vertical tip positioning during the acquisition time $t_{\text {acq }}$ of $6 \mathrm{~s}$ to a maximum deviation along $X(Z)$ of about $0.4 \mathrm{pm}(0.7 \mathrm{pm})$, respectively. This uncertainty is smaller than the grid spacing and, thus, negligible.

Frequency shift versus distance $\Delta f(z)$ curves are recorded by first approaching the tip from the tip-sample distance $z_{\text {ref }}$, as defined by the $\Delta f$ set point, closer to the surface to $z_{\min }$. The tip is then retracted along $Z$ to a maximum distance $z_{\max }$ while sampling the frequency shift $\Delta f$ data. The retraction (approach) curve is sampled when moving the tip from $z_{\min }$ to $z_{\max }\left(z_{\max }\right.$ to $\left.z_{\min }\right)$, respectively, and the tip is finally moved back to the reference height $z_{\text {ref }}$. The resulting $Z$ trajectory is depicted in Fig. 1(b). The stable point of smallest tip-sample separation $z_{\min }$ is highly dependent on the tip configuration and, accordingly, we chose it carefully for each experiment. Exemplary $\Delta f(z)$ and $F_{z}(z)$ curves are reproduced in Figs. 1(c) and $1(\mathrm{~d})$.

Our scan protocol requires a large number of parameters to be carefully chosen to optimize the data resolution, drift compensation accuracy, and signal to noise ratio. A list of parameters used for the data in this work is given in Table I.

\section{B. Force conversion}

Two of several force recovery strategies [28,29] have recently been compared by means of simulated data [30]. Based on this analysis, the Sader and Jarvis formalism [23] is expected to yield accurate results with reasonable numerical effort for the range of amplitudes used herein and is consequently employed. They found that the vertical interaction force $F_{z, t s}$ is given from an integration of the raw $\Delta f$ data:

$$
\begin{aligned}
F_{z, t s}(z)= & \frac{2 k}{f_{0}} \int_{z}^{\infty}\left[\left(1+\frac{\sqrt{A}}{8 \sqrt{\pi(q-z)}}\right) \Delta f(q)\right. \\
& \left.-\frac{A^{\frac{3}{2}}}{\sqrt{2(q-z)}} \frac{d(\Delta f(q))}{d q}\right] d q .
\end{aligned}
$$


TABLE I. Parameter list for the $Z X$ slice data acquisition.

\begin{tabular}{lcc}
\hline \hline parameter & variable & value \\
\hline Grid settings: & $p_{\mathrm{x}}$ & \\
Number of curves along $X$ & $p_{\mathrm{z}}$ & 201 \\
Points along $Z$ & $s_{\mathrm{x}}$ & 1000 \\
Size along $X$ & $s_{\mathrm{Z}}$ & $3.48 \mathrm{~nm}$ \\
Size along $Z$ & $\mathrm{res}_{\mathrm{x}}$ & $1.17 \mathrm{~nm}$ \\
Sampling resolution in $X$ & $\mathrm{res}_{\mathrm{Z}}$ & $17.3 \mathrm{pm}$ \\
Sampling resolution in $Z$ & & $1.2 \mathrm{pm}$ \\
Atom tracking: & $f_{\text {dither }}$ & \\
Dither frequency & $A_{\text {dither }}$ & $1.5 \AA$ \\
Dither amplitude & $t_{\text {track }}$ & $8 \mathrm{sz}$ \\
Tracking time & $t_{\text {update }}$ & $15 \mathrm{~min}$ \\
Drift comp. update interval & & \\
Data acquisition: & $B_{\mathrm{PLL}}$ & $400 \mathrm{~Hz}$ \\
PLL bandwidth & $t_{\text {sample }}$ & $3 \mathrm{~ms}$ \\
Time/sample along $Z$ & $t_{\text {acq }}$ & $6 \mathrm{~s}$ \\
Data acquisition time & & \\
\hline \hline
\end{tabular}

Here, $k$ is the cantilever stiffness, $f_{0}$ is the frequency of the free oscillating cantilever, $A$ is the oscillation amplitude and $z$ is the tip-sample distance.

Practically, the integration to infinity is replaced by a finite value $z_{\text {top }}$. This upper limit has to be chosen carefully by either a value larger than at least twice the oscillation amplitude $A$ plus the relevant interaction range, or by a value from which on $\Delta f$ remains zero to avoid artifacts in the resulting force. To maintain the high resolution close to the sample but to avoid unnecessary long measurement times in the long-range regime, we record several $\Delta f(z)$ curves at the end of every experiment with $z_{\text {top }}$ around $50 \mathrm{~nm}$. These data are combined with the site-specific, short-range grid curves by interpolating the longrange curves to match the sampling interval of the short-range data. Each curve resulting from this data combination spans more than twice the oscillation amplitude while at the same time maintaining the raw data across the interaction regime.

The integral in Eq. (1) is improper due to a pole of the integrand at $q=z$. The discrete formulation of Eq. (1) shown in Eq. (2) employs correction terms handling the pole as suggested in Ref. [31] (we implemented Eq. (2) in a MATLAB code):

$$
\begin{aligned}
F_{z, t s}\left(z_{k}\right)= & \frac{2 k}{f_{0}}\left[\Delta f\left(z_{k}\right) \Delta z+\frac{1 \sqrt{A}}{4 \sqrt{\pi}} \Delta f\left(z_{k}\right) \sqrt{\Delta z}\right. \\
& \left.-\frac{2 A^{\frac{3}{2}}}{\sqrt{2}} \frac{\Delta f\left(z_{k+1}\right)-\Delta f\left(z_{k}\right)}{\sqrt{\Delta z}}\right] \\
& +\frac{2 k}{f_{0}} \sum_{i=k+1}^{N-1}\left[1+\frac{\sqrt{A}}{8 \sqrt{\pi\left(z_{i}-z_{k}\right)}} \Delta f\left(z_{i}\right)\right. \\
& \left.-\frac{A^{\frac{3}{2}}}{\sqrt{2\left(z_{i}-z_{k}\right)}} \frac{\Delta f\left(z_{i+1}\right)-\Delta f\left(z_{i}\right)}{\Delta z}\right] \Delta z .
\end{aligned}
$$

We calibrated the oscillation amplitude $A$ using the constant- $\gamma$ method described by Heyde et al. [32]. The dynamic stiffness $k$ of the cantilever was obtained from the thermal cantilever noise as analyzed in detail by
TABLE II. Filter parameters used for the force recovery.

\begin{tabular}{lcc}
\hline \hline parameter & variable & value \\
\hline $\begin{array}{l}\text { Lanczos differentiator: } \\
\text { order }\end{array}$ & $n_{\mathrm{L}}$ & \\
$\begin{array}{l}\text { Savitzky-Golay filter: } \\
\text { degree } \\
\text { span }\end{array}$ & $m_{\mathrm{p}}$ & 5 \\
& $\begin{array}{l}n_{\mathrm{SG}} \\
\text { (corresponds to 7 pm) }\end{array}$ & 3 \\
\hline \hline
\end{tabular}

Lübbe et al. [33]. The resonance frequency $f_{0}$ of the free cantilever was determined by the phase-locked loop (PLL) using a high-quality reference oscillator.

All data were acquired at large PLL bandwidths and large pixel numbers. Digital filters are applied afterwards to reduce the noise in our measurement data. Specifially, Lanczos differentiators are employed for calculating the derivative in Eq. (2) and Savitzky-Golay filters are used for smoothing the data after the force recovery. For the latter, we use the smooth function implemented in MATLAB. This step allows us to critically check the filters influence and detect possible artifacts. To assess the quality of the force recovery, we use model calculations similar to the work by Welker et al. [30]. We evaluate and optimize the coefficient of determination $R^{2}$ as well as the deviation of the force minimum position $\Delta z_{\min }$ and value $\Delta F_{\min }$. Data are simulated from a Morse potential superimposed by random noise reflecting the experimental situation and tip-calcite interaction. From extensive simulations we are confident that we found most suitable parameters for the present experimental data as well as the noise level of our system. These parameters are listed in Table II. For these filters, experimental parameters and conditions we obtain an uncertainty in the total force in the order of $5 \mathrm{pN}$ due to the conversion.

\section{Short-range forces}

Lateral short-range forces $F_{y}$ are calculated from the negative derivative of the potential with respect to the horizontal direction,

$$
F_{y}=-\frac{\partial U}{\partial y}
$$

Due to the data alignment along the [42.1] direction (unit cell vector $\vec{b}$ ), we denote the lateral coordinate as $y$. We decreased the noise in the lateral force data by using a five-pixel mean filter along both axes.

The short-range vertical interaction forces $F_{z, \mathrm{SR}}$ are extracted by fitting and subtracting the long-range van der Waals forces $F_{\mathrm{vdW}}$ from our total force $F_{z}$ data. This procedure is a delicate task [24,34], especially as the herein investigated calcite system does not allow for using the reference-curve based approach $[34,35]$. We do not include electrostatic interactions as we experimentally minimized electrostatic forces using the Kelvin probe technique measured at a representative sample position before the slice data acquisition [36].

Based on a detailed analysis [24] of ten different van der Waals descriptions, we identified the van der Waals force law introduced by Zanette et al. [37] to model the van der Waals 

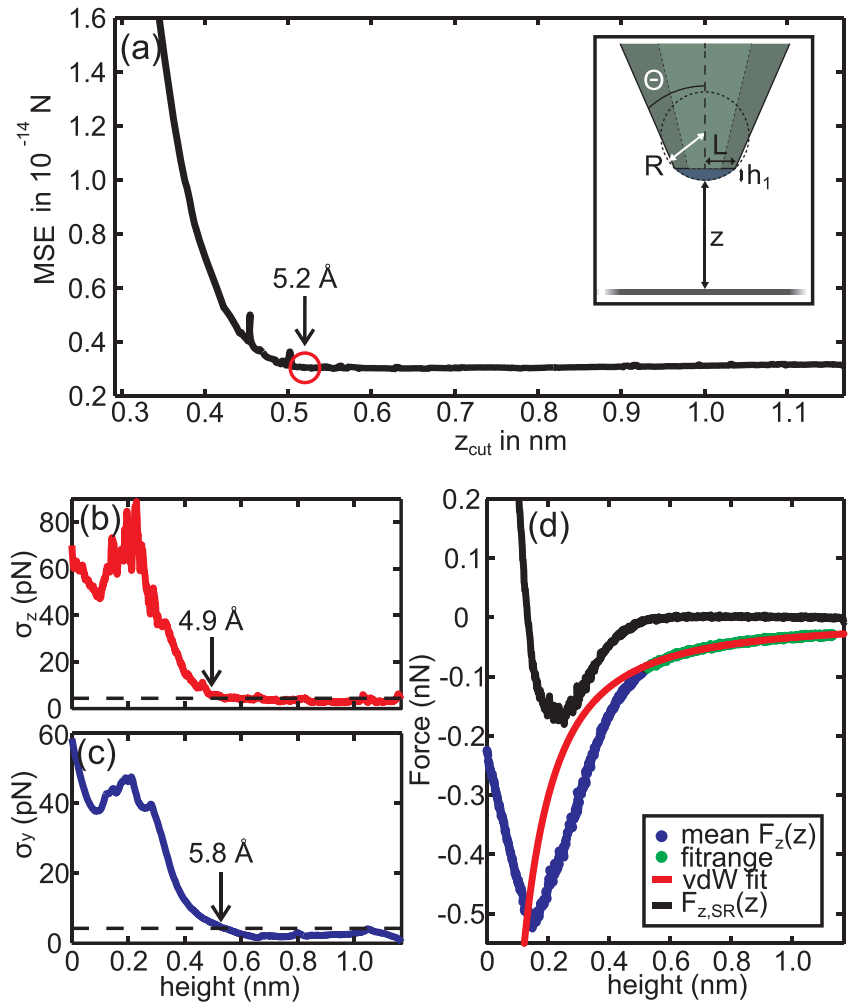

FIG. 2. (Color online) (a) Fit quality expressed as the mean squared error MSE from fitting a van der Waals model to the mean experimental curve as a function of the lower fit range $z_{\text {cut }}$. (b) Standard deviation $\sigma_{z}$ of the vertical force $F_{z}$. (c) Standard deviation $\sigma_{y}$ of the lateral force $F_{y}$. Resulting cutoff points are marked in both panels. (d) Mean total vertical interaction force $\overline{F_{z}}$ (blue), van der Waals interaction force $F_{\mathrm{vdW}}$ (red), and resulting mean vertical short-range force $\overline{F_{z, \text { SR }}}$ (black) (Ref. [24]).

contribution most reliably for our data. The central challenge of short-range force extraction is to find the cutoff point $z_{\text {cut }}$, which marks the transition from pure long-range and siteunspecific to mixed interaction when bringing the tip closer to the surface.

Results of our analysis are reproduced in Fig. 2, where panel (a) presents the quality of the fit using the mean squared error MSE as a function of $z_{\text {cut }}$. The best regression is found when fitting the data along the $z$ range $\left[z_{\text {cut }}, z_{\max }\right]$ with $z_{\text {cut }}=5.2 \AA$. We introduced two further independent criteria for refining the value of $z_{\text {cut }}$ [24], namely the standard deviation $\sigma$ of vertical and lateral forces. In presence of site-specific forces, a clear increase in $\sigma_{z}\left(\sigma_{y}\right)$ of the vertical (lateral) force is expected. The results of this analysis are shown in Figs. 2(b) and 2(c) and allows us to refine $z_{\text {cut }}$ to $5.8 \AA$.

The mean total vertical force $\overline{F_{z}}$, the modelled van der Waals interaction $F_{\mathrm{vdW}}$ and the resulting mean short-range force $\overline{F_{z, \mathrm{SR}}}$ using $z_{\mathrm{cut}}=5.8 \AA$ are depicted blue, red and black, respectively, in Fig. 2(d). For the full data set, $F_{\mathrm{vdW}}$ is subtracted from every $F_{z}(y)$ curve. Further details and the resulting model parameters are given in Appendix B. We find an uncertainty of the force minimum position and magnitude of about $\delta z=0.4 \AA$ and $\delta F=60 \mathrm{pN}$, respectively. Additionally, the position of the $F_{z, \text { SR }}$ zero, i.e., the transition from attractive to repulsive forces, is subject to an uncertainty of about $\delta z=0.4 \AA$.

\section{PROPERTIES OF CALCITE}

Calcite(10.4) is the energetically most favorable surface of the most stable polymorph of calcium carbonate $\left(\mathrm{CaCO}_{3}\right)$. Calcite crystallizes in a trigonal crystal system described by space group $R \overline{3} c$ [38], the hexagonal bulk unit cell is depicted in Fig. 3(a).

The bulk-truncated, unreconstructed surface of calcite(10.4) has a rectangular unit cell with dimensions [39] of $4.99 \times 8.10 \AA^{2}$ and with the unit cell vectors $\vec{a}$ and $\vec{b}$ oriented along the [01.0] and [ $\overline{42.1]}$ crystallographic directions, respectively. Two calcium ions and two carbonate $\left(\mathrm{CO}_{3}\right)$ groups are located within every unit cell, the two carbonate groups being tilted in the bulk by an angle of $44.63^{\circ}$ with respect to the (10.4) planes [see Fig. 3(b)] [15,39]. Additionally, these two carbonate groups are rotated with respect to each other, causing the topmost oxygen atom to point either to the left or to the right in a zigzag fashion [see Fig. 3(b)].

It is mainly the tilt of the carbonate groups, as visualized by a green dashed line in Fig. 3(b), which reduces the symmetry of the surface and renders it a member of the plane symmetry group $p g$. Only one glide plane reflection with the glide axis oriented along the [42.1] direction is left as a surface

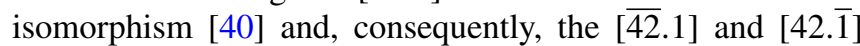
directions are not equivalent. We note that the undetermined surface orientation is fully defined by the tilt direction of the carbonate groups.

Figure 3(c) presents one prototypical contrast reflecting the surface unit cell and the zigzag pattern of the topmost oxygen atoms [16]. Two reconstructions, which have been observed before and whose existence and origins are still discussed controversially [41-44], are not resolved in this contrast mode. We note in this context that numerous different NC-AFM contrast modes have been observed on calcite(10.4) before [16]. Although the general alignment of the [01.0]

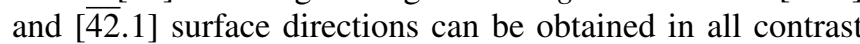
modes directly from the unit cell size ${ }^{1}$ in $X Y$-scanned images, the absolute surface orientation remains, as mentioned above, unknown as the tilt of the carbonate groups is first hand not revealed within these data [see Figs. 3(d) and 3(e) for the two possibilities].

\section{OPTICAL IDENTIFICATION}

Besides its natural relevance, calcite constitutes a particularly well-suited sample for relating the surface asymmetry measured within this work on the microscopic scale to the crystal directions, as this sample provides an independent way of easily identifying the surface orientation by macroscopic means without the necessity of, i.e., x-ray diffraction experiments. An analysis of the optical birefringence of the bulk

\footnotetext{
${ }^{1}$ Besides a careful calibration of the microscope, the images are corrected for thermal drift [54] and scanner creep to accurately determine the unit cell sizes.
} 
(a)

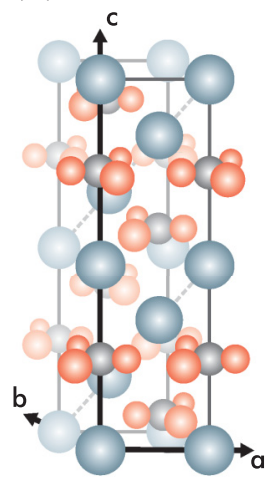

(b)

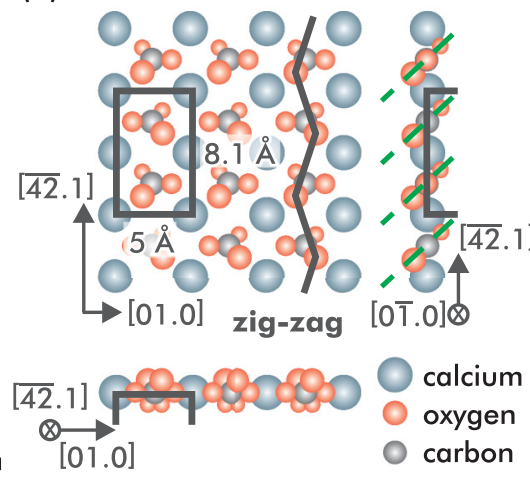

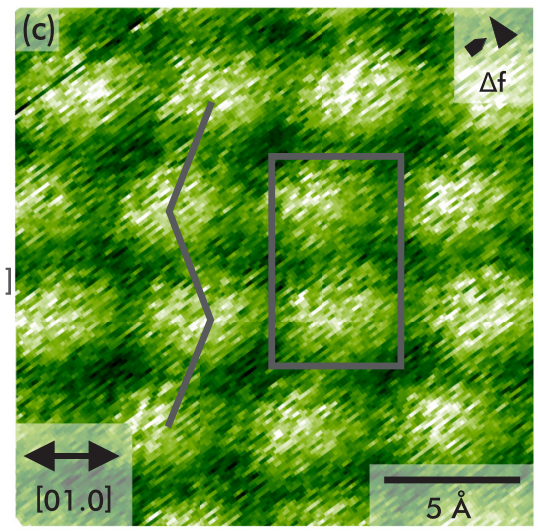

(d)

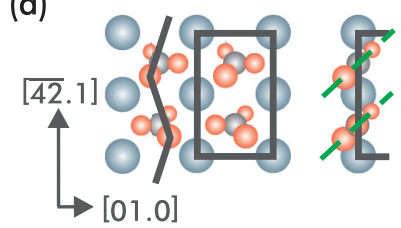

(e)

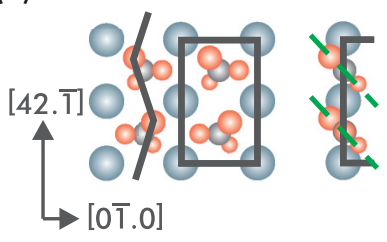

FIG. 3. (Color online) (a) Hexagonal bulk unit cell of calcite and (b) model of the (10.4) surface. The tilt of the carbonate groups is visualized by dashed green lines. (c) Typical NC-AFM image representing the zigzag of the protruding oxygen atoms [16]. From measuring the unit cell dimensions, an ambiguity in determining the absolute calcite surface orientation remains. (d) and (e) Two different possibilities leaving the tilt of the carbonate groups unknown.

material reveals that the (10.4) projected split vector $\vec{p}$ between the ordinary and extraordinary ray proceeds along the [42.1. $]$ direction (see Appendix A for the full derivation) as it is defined by

$$
\begin{aligned}
\vec{p} & =-\frac{2 \sqrt{3} a c t\left(\epsilon_{1}-\epsilon_{3}\right)}{12 a^{2} \epsilon_{3}+c^{2} \epsilon_{1}} \vec{e}_{b} \\
& \approx-0.11 t \vec{e}_{b} .
\end{aligned}
$$

Here, $a$ and $c$ are the bulk lattice constants of calcite, $\epsilon_{1}$ and $\epsilon_{3}$ the dielectric constants for the ordinary and extraordinary ray, respectively, $t$ is the crystal thickness and $\vec{e}_{b}$ is a normalized unit vector pointing along the [42.1] direction. The relation between this spilt vector, the wave vector, and the Poynting vectors is sketched in Fig. 4(a) and derived in Appendix A.

Photographic pictures of a calcite sample mounted inside the sample holder are shown in Figs. 4(b) to 4(d). A printed black cross is brought beneath a crystal of thickness $t \sim 8 \mathrm{~mm}$. Figures 4(c) and 4(d) were acquired with a linear polarizing filter in front of the camera lens, the linear polarizing direction differs by $90^{\circ}$ and is indicated in each lower right corner. In Fig. 2(c), the picture of the underlying cross caused by the ordinary ray is revealed, while panel (d) shows the picture due to the extraordinary ray. The split vector $\vec{p}$ points to the lower left corner, and thus, determines the [42. $\overline{1}]$ vector pointing into this direction.

\section{TIP-SAMPLE FORCE MEASUREMENT}

In order to microscopically determine the surface orientation and to further understand the tip-calcite interactions, we extend the force measurement to the vertical dimension with results in the form of a $Z Y$-slice from the calcite(10.4) surface presented in Fig. 5. These data were acquired using the atom-tracking technique [21,22] for drift compensation and a systematic sampling of the data space as described before. The $z$-dependent frequency-shift $\Delta f(z)$ data [Fig. 5(a)] were acquired along a horizontal line as is indicated in the inset. Vertically, the relevant regime of tip-sample distances up to about $1.2 \mathrm{~nm}$ is reproduced, only site-unspecific long-range forces are measured at larger $z$. The total vertical forces $F_{z}$
[Fig. 5(b)] are calculated using the Sader-Jarvis formalism [23] and the short-range vertical forces $F_{z, \text { SR }}$ [Fig. 5(c)] are extracted from the van der Waals background subtraction [24]. A red (blue) color depicts an attractive force on the tip to the right (left), respectively, in the lateral forces $F_{y}$ [Fig. 5(d)]. The positive horizontal axis points along the $[42 . \overline{1}](y)$ calcite direction as determined from the optical criterion for this specific crystal. A tiny misalignment of these data with respect to the axis is apparent from the lateral force data, where a slight decrease in the overall contrast is apparent. The difference appears to be too small to be detectable in the vertical forces.
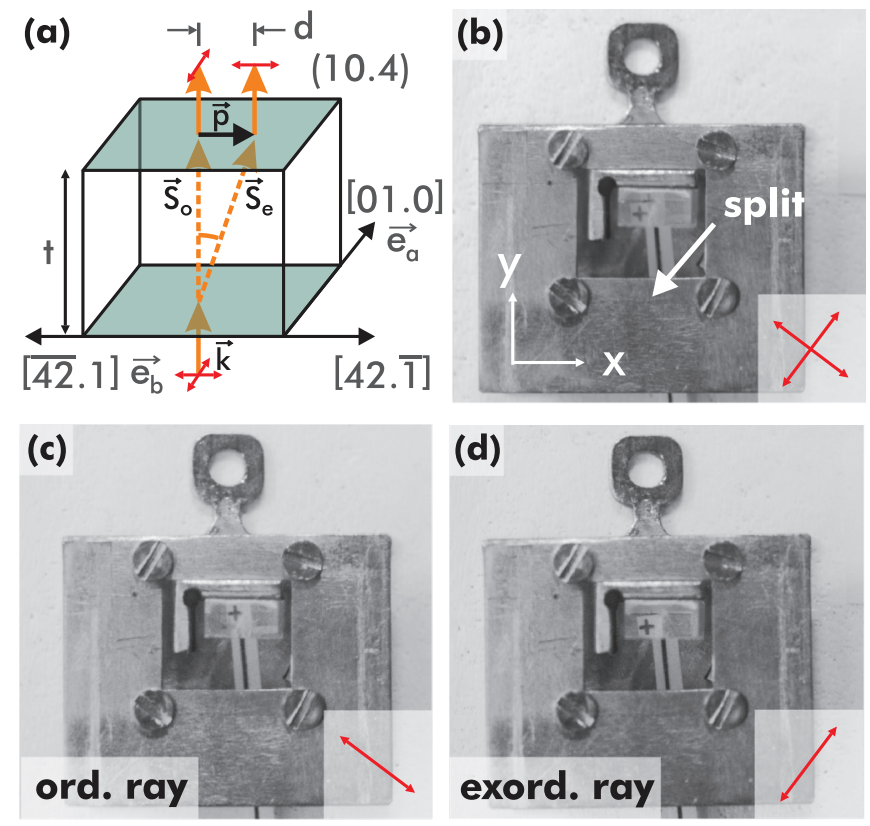

FIG. 4. (Color online) Macroscopic method to determine the absolute surface orientation. (a) The split vector $\vec{p}$ between the Poynting vectors $\vec{S}_{\text {o }}$ and $\vec{S}_{\text {e }}$ in the (10.4) surface plane points along the [42.1] direction. This split vector is easily identifiable by determining the ordinary and extraordinary ray using a linear polarizing filter, see panels (c) and (d). 

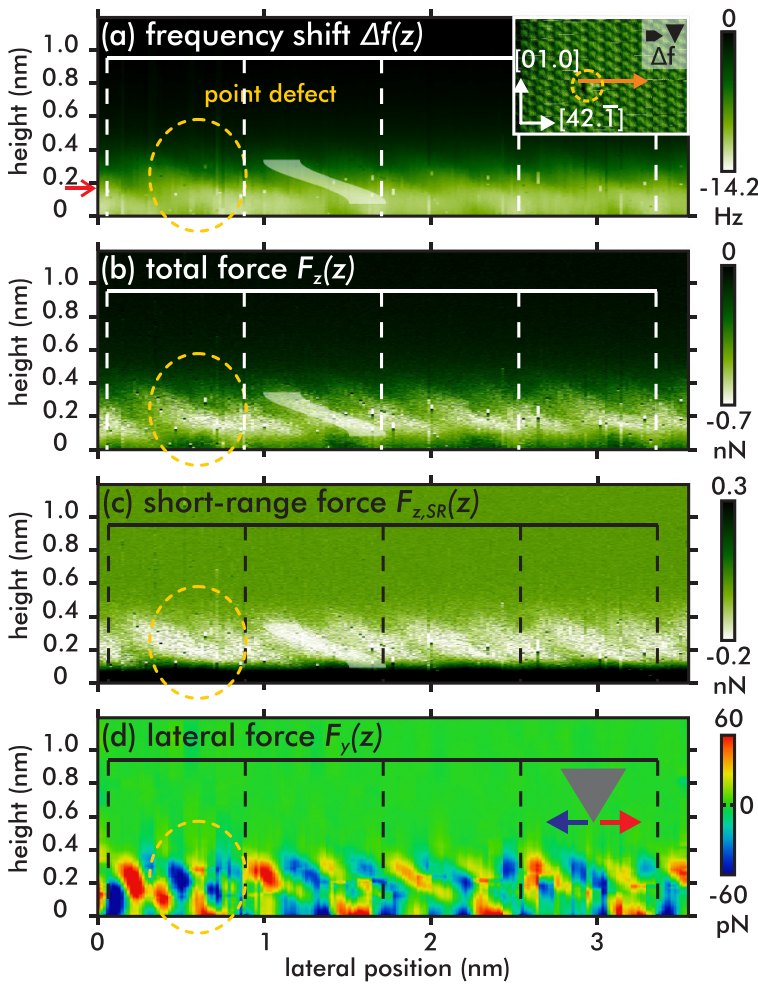

(e)

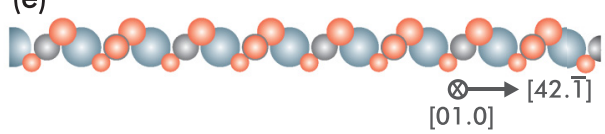

FIG. 5. (Color online) (a) Frequency shift $\Delta f$ data from a $Z Y$ dataset with size $1.17 \times 3.48 \mathrm{~nm}^{2}$ (total of $1000 \times 201$ pixel). The inset shows the $10 \times 6.7 \mathrm{~nm}^{2} X Y$-scanned $\Delta f$ image prior and subsequent to the $2 \mathrm{D}$ data acquisition. The orange arrow indicates the position of the $Z Y$ measurement, the yellow circle marks a point defect. The $z$ position of this inset is marked by a red arrow at the vertical axis of the $Z Y$ data set. (b) Total vertical interaction forces $F_{z}$ calculated from (a). (c) Short-range vertical forces $F_{z, \mathrm{SR}}$ and (d) lateral forces. Unit cell periodicity is marked in all images and the half-transparent lines guide the eye to the tilted features. (e) True to scale calcite(10.4) surface model aligned according to long-range attractive interactions with the $\mathrm{CO}_{3}$ groups.

The total force is overall attractive (negative) throughout this data set with the minimum at about $-0.6 \mathrm{nN}$.

The capability of imaging a single defect (marked by dashed yellow circles) indicates the presence of a most sharp AFM tip. The position of the $Z Y$ data slice was chosen such that it partly covers this surface defect. The difference in corrugation within each unit cell is ascribed to the zigzag orientation of the carbonate groups. As is apparent from the model in Fig. 3(b), every second top-most oxygen atom points out of the plane mapped in the experiment, while every other points inwards. This modulation is apparent in both, vertical and lateral data.

The most striking observation in Figs. 5(a) to 5(d), however, is a significant asymmetry observed in the raw frequency shift $\Delta f$ and force data sets. When following either a bright or dark region along the $Z$ axis, a bend of the respective regions upon approach to the surface becomes apparent in panels (a) to (c). We include a half-transparent guide to the eye for illustrating this tilt. It manifests a clear asymmetry along the vertical axis in agreement with the surface asymmetry along this direction caused by the tilt of the carbonate groups.

We critically analyzed the NC-AFM setup and data processing (including the relative tip-sample orientation, cantilever tilt, piezo artifacts and force recovery strategy) and found the same orientation of the tilt in repeated experiments under different tip and sample conditions. Consequently, we are confident about a physical origin of the observed tilt and identify two sources: the tilt is supposedly caused by either an asymmetry of the investigated surface or of the scanning tip. An asymmetric tip apex can lead to an asymmetry in the tip-sample interaction forces, which might propagate either directly or via a tip or a surface relaxation into the measured data. This has been observed before on high-symmetry surfaces, namely on $\mathrm{KBr}(001)$ [10,45], $\mathrm{NaCl}(001)$ [11], and graphite(0001) [46]. Any measured asymmetric tilt of the surface species on these substrates is in clear disagreement with the surface geometry and symmetry properties. Consequently, the data have been explained by either a tip deformation or by a tip-induced sample manipulation upon close approach due to an asymmetric tip.

\section{DISCUSSION}

Foster et al. modeled the calcite surface structure in presence of a scanning probe tip and found a surface relaxation including shifts of all surface species as well as a rotation of the surface carbonate groups [12,47]. Their numerical calculations have furthermore suggested two possibilities for the tip-surface interaction [12]: a more attractive force between the AFM tip and either the calcium or the carbonate ion, causing the carbonate group being either pushed into or pulled outwards from the surface by the atop positioned tip. Our data are in agreement with the latter situation, namely a larger attractive interaction between the tip and the carbonate ion. First, we observe at small tip-sample distances in $X Y$ images a zigzag structure [see inset Fig. 5(a)] that has been associated with the alternating orientation of the protruding oxygen atoms before $[12,48]$. Second, the high-symmetric calcium atoms are unlikely to cause a tilted feature. Third, this assignment would, in a straightforward electrostatic picture, be in agreement with a positive tip termination. This situation has been postulated before as one imaging mode for the calcite(10.4) surface [12,47] and has also been suggested as a general property of conducting tips [49].

Following this conclusion, an analysis of the transition from site-unspecific long-range interactions to the site-specific short-range interaction regime in the total vertical force $F_{z}$ data allows for assigning the surface species. Figure 6(a) presents a line profile from this regime, extracted at $z=4.2 \AA$ and averaged over $0.59 \AA$ [see Fig. 6(b)]. A sinusoidal function is fitted to the experimental data (in red) as a guide to the eye. At this tip-sample distance, we expect to measure the surface structure mainly undisturbed by the presence of the tip and, thus, can use this region to relate our experimental data to a model of the surface structure.

If we assume an attractive interaction of the tip with the full carbonate group as substantiated before, we can assign the positions of maximum attractive interaction to the positions of 


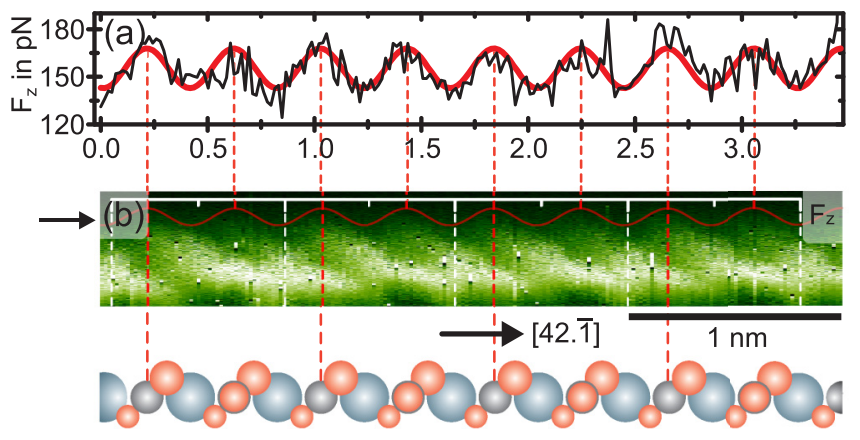

FIG. 6. (Color online) Onset of total vertical forces $F_{z}$ employed for the assignment of surface species in the data set. The line profile in (a) is extracted at the vertical onset of atomic corrugation at $z=4.2 \AA$ from the $F_{z}$ data in (b).

the carbonate group centers as depicted in Fig. 6. Interestingly, this assignment is in agreement with the measurement of lateral forces presented later in Fig. 7(b). We find a sector towards which the tip feels an attractive force from both the left and the right sides in the lateral forces. This sector is located inbetween the red and blue area. Importantly, these areas of
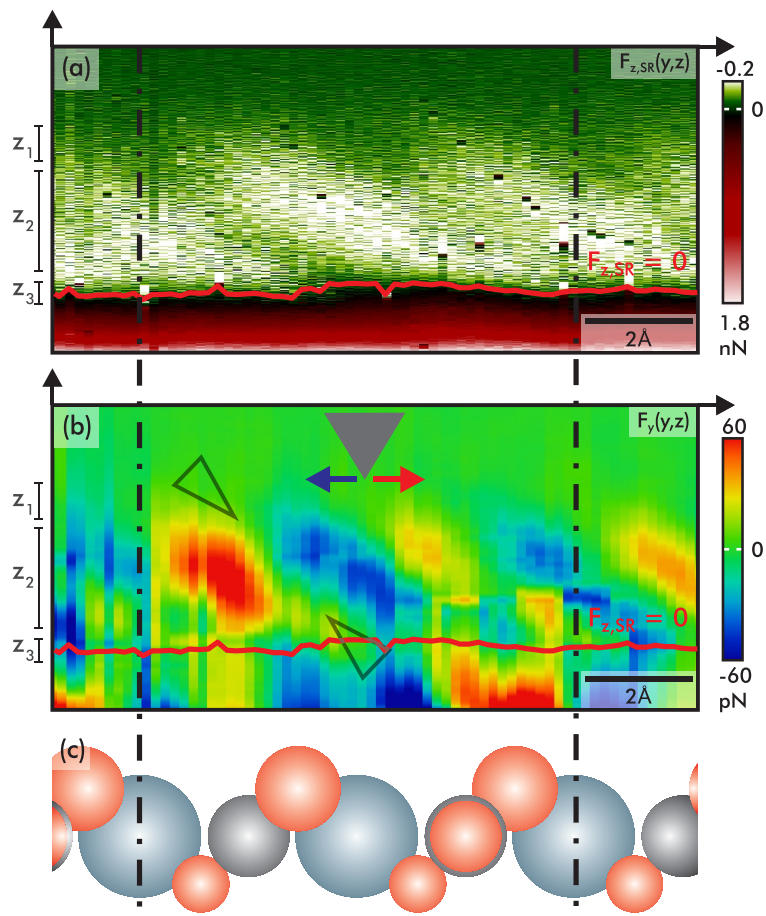

I

$(x)[01.0] \otimes \longrightarrow[42 . \overline{1}](y)$

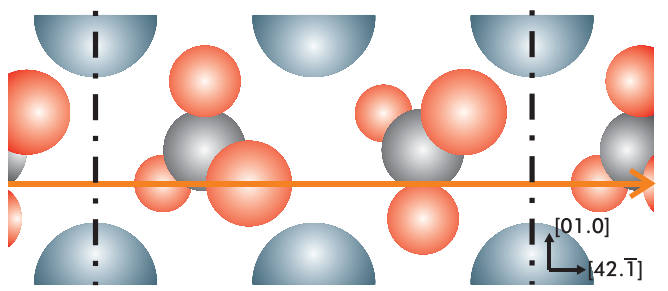

FIG. 7. (Color online) (a) Vertical short range forces $F_{z, \text { SR }}$ and (b) lateral forces $F_{y}$ extracted for a single surface unit cell. The heights of $F_{z, \mathrm{SR}}(z)=0$ are marked by red lines. (c) Corresponding model in side- and top-view aligned with the experimental data. large lateral forces are clearly tilted and this tilt has the same orientation as observed in the vertical forces before.

Thus, these data are in full agreement with imaging the carbonate groups attractive and as a single entity at large tipsample distances (height $z_{1}$ as indicated in Fig. 7). The surface structure in Figs. 5 and 7 are accordingly included.

To further analyze the tip-surface interaction mechanism on the calcite(10.4) surface, we focus on the vertical $F_{z, \text { SR }}$ and lateral $F_{y}$ short-range forces across a single unit cell extracted from the full dataset and reproduced in Figs. 7(a) and 7(b), respectively. The short-range forces $F_{z \text {,SR }}$ describe the specific interaction of the tip front atoms with each surface species. We determine the tip height for the optimum binding position from the zero of $F_{z, \mathrm{SR}}$ (simultaneously the minimum of the short-range interaction potential) and mark these heights $z_{3}$ by a solid red line in Figs. 7(a) and 7(b). At the same height, the lateral forces exhibit a regime of reduced interactions-a finding giving us strong confidence about the validity of our short-range force extraction procedure.

Based on this analysis, the tilt is exclusively observed at tip-sample distances larger than the force zero, namely, at heights $z_{1}$ and $z_{2}$ as marked in Fig. 7. This allows us to conclude that purely attractive short-range interactions between the tip apex and the surface species are responsible for the observed tilt. This tilt constitutes the passage from a short-range force uninfluenced carbonate group (at tip-sample distances $z>z_{1}$ ) to the optimum carbonate binding situation in the combined tip-sample potential (at tip-sample distance $z=z_{3}$ ).

When uniting all findings, we explain the orientation of the tilt in the regime of attractive short-range forces by a transition between two interactions: at large tip-sample distances (around height $z_{1}$ in Fig. 7) our $F_{z, \text { SR }}$ data suggests the largest attraction between the tip and the carbonate group as one entity. When reducing the tip-sample distance at $z<z_{1}$, we explain the tip-surface attraction being dominated by the force between the tip and the protruding oxygen atom only; the proximity to the oxygen atom is understood to outweigh the attraction to the whole group. Both cases are illustrated in Fig. 8. This explanation is further substantiated when inspecting the lateral force $F_{y}$ data in Fig. 7(b): a zone (marked by two triangles) to which the tip is dragged from both the left and the right sides is clearly apparent. From the surface structure depicted below, we identify the topmost oxygen atom lying centered in this zone. With this explanation, the observed shift of the tilt to the right originates in the position of the oxygen atom and, thus, in the orientation of the carbonate group and the surface. (a)

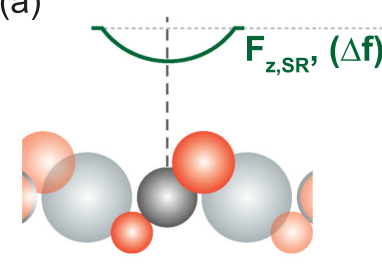

(b)

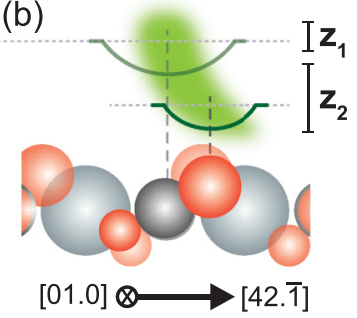

FIG. 8. (Color online) (a) The full carbonate group is imaged at large tip-sample distances, while (b) the maximum attractive interaction shifts towards the topmost oxygen atom, located at the right. 
The trajectory of the tilt in the lateral and vertical force data within height ranges $z_{1}$ and $z_{2}$ is in good agreement with the surface geometry. However, relaxation of the surface carbonate group or secondary interactions with the neighboring surface or tip species could lead to an amplification of the observed tilt, especially giving a plausible explanation for the tail observed in the vertical interaction at tip-sample distances $z_{3}$ and closer. The relaxation of single molecules has very recently been measured for the case of two CO molecules [50], which bend towards each other in the attractive interaction regime. The observation of strikingly similar features in our lateral force data leads us to the conclusion that a relaxation of the carbonate group in the surface might amplify the interaction transition, especially at small tip-sample distances. The repeatability of observing the same tilt orientation under different conditions, the identification of pure attractive interactions and our explanation gives us strong confidence that the calcite surface is the source for the tilt orientation in our data and, thus, allows for determining the absolute surface orientation.

\section{CONCLUSIONS}

In conclusion, we investigated the tip-sample interaction forces on a low-symmetry substrate, namely the calcite(10.4) surface. We found a pronounced tilt of the measured surface species in our vertical and lateral force data, which we clearly identified as pure attractive short-range interaction. We explain these attractive short-range force data by a transition from the tip interacting with the entire carbonate group to an interaction with the topmost oxygen atom only at reduced tip-sample distances. The experimentally observed tilt is related to the surface structure and symmetry and discloses the absolute surface orientation - a parameter especially relevant when studying physicochemical processes in the context of, i.e., understanding and mimicking biomineralisation. Thus, the ability to directly determine an essential surface property from interaction force data constitutes another important step in the field of investigating crystalline surfaces in real space.

\section{ACKNOWLEDGMENTS}

This work was supported by the German Research Foundation through grant KU 1980/4-1 and by a Grant-in-Aid for Scientific Research from the Ministry of Education, Culture, Sports, Science and Technology of Japan (26110516, 26600015, 26600099, 25106002, 24360016, and 22221006), and Funding Program for Next Generation World-Leading Researchers. P.R. gratefully acknowledges funding from the Alexander von Humboldt Foundation. We thank Ralf Bechstein for stimulating discussions and Jürgen Köble (Omicron Nanotechnology) for helpful support with the MATE scripting interface.

\section{APPENDIX A: MACROSCOPIC IDENTIFICATION OF THE ABSOLUTE SURFACE ORIENTATION OF CALCITE}

Calcite offers an elegant strategy to determine the absolute (10.4) surface orientation macroscopically by exploiting the birefringence of the material. This appendix presents the derivation for relating the [42.1] orientation to the split direction of the ordinary and extraordinary ray in the (10.4) plane for a light ray passing perpendicular through the (10.4) surface.

We first define the matrix $\hat{\mathbf{M}}$ as the transformation matrix between the hexagonal Miller indices $\vec{r}_{h}$ used in crystallography for calcite and the cartesian coordinates $\vec{r}$ used to describe the optical properties from $\vec{r}=\hat{\mathbf{M}} \vec{r}_{\mathrm{h}}$ as

$$
\hat{\mathbf{M}}=\left(\begin{array}{ccc}
a & -\frac{a}{2} & 0 \\
0 & a \frac{\sqrt{3}}{2} & 0 \\
0 & 0 & c
\end{array}\right)
$$

with $a$ and $c$ being the bulk lattice constants of calcite [39]. In the following, all coordinates given refer to the cartesian system unless noted by a lower index $h$. The normalized unit cell vectors of the (10.4) surface in a Cartesian coordinate system are given by

$$
\vec{e}_{a}=\hat{\mathbf{M}}\left(\begin{array}{l}
0 \\
1 \\
0
\end{array}\right)_{\mathrm{h}}=\frac{1}{2}\left(\begin{array}{c}
-1 \\
\sqrt{3} \\
0
\end{array}\right)
$$

and

$$
\vec{e}_{b}=\hat{\mathbf{M}}\left(\begin{array}{c}
-4 \\
-2 \\
1
\end{array}\right)_{\mathrm{h}}=\frac{1}{\sqrt{12 a^{2}+c^{2}}}\left(\begin{array}{c}
-3 a \\
-\sqrt{3} a \\
c
\end{array}\right) .
$$

The normalized wave vector $\vec{k}$ for a plane wave entering the calcite crystal perpendicular through any (10.4) plane is given by

$$
\vec{k}=\frac{\vec{e}_{\mathrm{a}} \times \vec{e}_{\mathrm{b}}}{\left|\vec{e}_{\mathrm{a}} \times \vec{e}_{\mathrm{b}}\right|}=\frac{1}{2 \sqrt{12 a^{2}+c^{2}}}\left(\begin{array}{c}
\sqrt{3} c \\
c \\
4 \sqrt{3} a
\end{array}\right) .
$$

We define $\theta$ as the angle between the negative wave vector $\vec{k}$ and the $z$ axis $\vec{e}_{\mathrm{z}}$. This angle is identical to the well-known angle of the carbonate group tilt in the (10.4) surface plane and is calculated to

$$
\theta=\angle\left(-\vec{k}, \vec{e}_{\mathrm{z}}\right) \approx 44.63^{\circ} .
$$

This equivalency is visualized in Fig. 9 (note that the wave vector $\vec{k}$ and the vector $\vec{e}_{(10.4)}$ normal to the (10.4) surface are antiparallel).

Calcite is optically uniaxial. The electric permittivity tensor $\hat{\epsilon}$ has in the principal coordinate system following diagonal quadratic form:

$$
\hat{\epsilon}=\left(\begin{array}{ccc}
\epsilon_{1} & 0 & 0 \\
0 & \epsilon_{1} & 0 \\
0 & 0 & \epsilon_{3}
\end{array}\right) .
$$

Here, $\epsilon_{1}$ and $\epsilon_{3}$ are the electric permittivities for the principal axes. The relation $n=\sqrt{\epsilon}$ generally links the indices of refraction to the electric permittivities for the optical regime and we use values from Ref. [51],

$$
\begin{aligned}
& \epsilon_{1}=n_{\mathrm{o}}^{2} \approx 2.749, \\
& \epsilon_{3}=n_{\mathrm{e}}^{2} \approx 2.208 .
\end{aligned}
$$




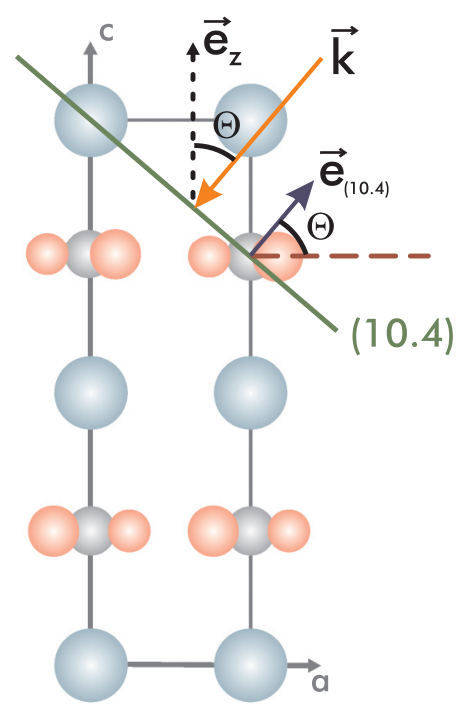

FIG. 9. (Color online) Relation between the wave vector $\vec{k}$, the unit cell vector $\vec{e}_{z}$ along $c$, the surface normal vector $\vec{e}_{(10.4)}$ and the angle $\Theta$. Note that for simplicity only the projection to the $a-c$ plane is shown.

For our specific case of light rays entering the crystal not along a principal axis, but perpendicular to the (10.4) surface, we find the refractive index for the extraordinary ray using formula 6.3-15 from [52]:

$$
\begin{gathered}
\frac{1}{n_{\mathrm{e}}(\theta)^{2}}=\frac{\cos (\theta)^{2}}{n_{\mathrm{o}}^{2}}+\frac{\sin (\theta)^{2}}{n_{\mathrm{e}}^{2}}, \\
n_{e}(\theta) \approx 1.57,
\end{gathered}
$$

where $\theta$ is given by Eq. (A5). The refractive index $n_{\mathrm{o}} \approx 1.66$ for the ordinary ray remains unchanged irrespective of the ray orientation.

Equation (A9) has been derived for optical uniaxial materials using the optical indicatrix as the representation of $\hat{\eta}=\hat{\epsilon}^{-1}$. This optical indicatrix can be visualized by an ellipsoid as depicted in Fig. 10(a). The intersection of the (10.4) plane with this ellipsoid is defined as the index ellipse and is presented in Fig. 10(b). (a)

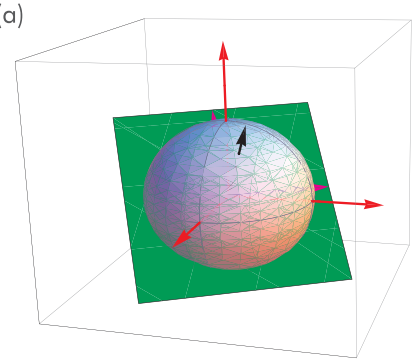

(b)

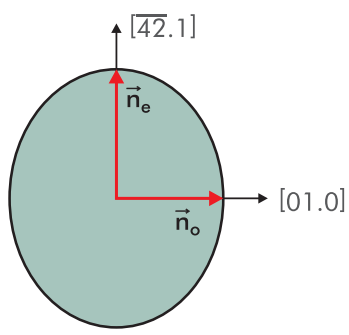

FIG. 10. (Color online) (a) Optical indicatrix for $\mathrm{CaCO}_{3}$ (blue sphere) including the position of the (10.4) surface plane (green plane). Base vectors of a Cartesian coordinate system (in red) and unit cell vectors of the (10.4) plane (in magenta) are included. (b) Index ellipse for a light ray crossing the (10.4) plane perpendicular.
The semiminor (semimajor) axis in this index ellipse defines the indices of refraction $n_{\mathrm{o}}\left(n_{\mathrm{e}}\right)$ for the ordinary (extraordinary) ray, respectively. We will show later that the semiminor (semimajor) axis is furthermore oriented along the [01.0] ([42.1]) directions, respectively. The latter statement also holds for the electric displacement field vector $\vec{D}_{\mathrm{o}}\left(\vec{D}_{\mathrm{e}}\right)$, respectively.

The normalized Poynting vector for both, the ordinary and extraordinary ray, can be expressed by

$$
\vec{S}_{i}=\frac{\vec{E}_{i, 0} \times\left(\vec{k} \times \vec{E}_{i, 0}\right)}{\left|\vec{E}_{i, 0} \times\left(\vec{k} \times \vec{E}_{i, 0}\right)\right|},
$$

where $\vec{E}_{i, 0}$ is the electric field vector and $\vec{k}$ is the wave vector. For light rays entering the crystal perpendicular to the (10.4) planes, the wave vector is oriented perpendicular to the (10.4) planes as given by Eq. (A4). In the following, we set $i=0$ $(i=\mathrm{e})$ for the ordinary (extraordinary) ray, respectively.

\section{Ordinary ray}

The electric displacement vector $\vec{D}_{\text {o }}$ for the ordinary ray is normal to the plane defined by the optical axis (here $\vec{e}_{z}$ ) and the direction of wave propagation $\vec{k}$ [52]. Thus, we can calculate $\vec{D}_{\text {o directly from }}$

$$
\vec{D}_{\mathrm{o}}=\frac{\vec{e}_{z} \times \vec{k}}{\left|\vec{e}_{z} \times \vec{k}\right|}=\frac{1}{2}\left(\begin{array}{c}
-1 \\
\sqrt{3} \\
0
\end{array}\right) .
$$

The vector is normalized to one. It is directly apparent that this vector is identical to the [01.0] unit cell vector $\vec{e}_{a}$ [Eq. (A2)]. We find the according electric field vector $\vec{E}_{\mathrm{o}}$ from $\vec{E}_{\mathrm{o}}=\hat{\epsilon}^{-1} \vec{D}_{\mathrm{o}}$ and finally calculate the normalized Poynting vector from Eq. (A11) to

$$
\vec{S}_{\mathrm{o}}=\frac{1}{2 \sqrt{12 a^{2}+c^{2}}}\left(\begin{array}{c}
\sqrt{3} c \\
c \\
4 \sqrt{3} a
\end{array}\right) .
$$

This vector is identical to the wave propagation vector $\vec{k}$.

\section{Extraordinary ray}

For the extraordinary ray, the electric displacement vector $\vec{D}_{\mathrm{e}}$ is normal to the wave propagation vector $\vec{k}$ and lies in the plane spanned by $\vec{k}$ and $\vec{e}_{z}[52]$. These relations are formulated by

$$
\begin{aligned}
& 0=\vec{D}_{\mathrm{e}} \cdot\left(\vec{k} \times \vec{e}_{z}\right), \\
& 0=\vec{D}_{\mathrm{e}} \cdot \vec{k} .
\end{aligned}
$$

We further normalize $\vec{D}_{\mathrm{e}}$ by $\left|\vec{D}_{\mathrm{e}}\right|=1$ and ensure its orientation along the positive $z$ axis from $0<\vec{D}_{\mathrm{e}} \cdot \vec{e}_{z}$. The normalized electric displacement vector $\vec{D}_{\mathrm{e}}$ is found as the solution of these equations to

$$
\vec{D}_{\mathrm{e}}=\frac{1}{\sqrt{12 a^{2}+c^{2}}}\left(\begin{array}{c}
-3 a \\
-\sqrt{3} a \\
c
\end{array}\right) .
$$


This vector is aligned along the [ $\overline{42} .1]$ direction as it is identical with the unit cell vector $\vec{e}_{b}$ [Eq. (A3)]. The corresponding electric field vector is calculated from $\vec{E}_{\mathrm{e}}=\hat{\epsilon}^{-1} \vec{D}_{\mathrm{e}}$ and we finally find the normalized Poynting vector for the extraordinary ray:

$$
\vec{S}_{\mathrm{e}}=\frac{1}{2 \sqrt{12 a^{2} \epsilon_{3}^{2}+c^{2} \epsilon_{1}^{2}}}\left(\begin{array}{c}
\sqrt{3} c \epsilon_{1} \\
c \epsilon_{1} \\
4 \sqrt{3} a \epsilon_{3}
\end{array}\right) .
$$

\section{Split vector}

The angle $\alpha$ between the two Poynting vectors is given by

$$
\begin{aligned}
\alpha & =\arccos \left(\frac{\vec{S}_{\mathrm{o}} \cdot \vec{S}_{\mathrm{e}}}{\left|\vec{S}_{\mathrm{o}}\right|\left|\vec{S}_{\mathrm{e}}\right|}\right) \\
& =\arccos \left(\frac{12 a^{2} \epsilon_{3}+c^{2} \epsilon_{1}}{\sqrt{\left(12 a^{2}+c^{2}\right)\left(12 a^{2} \epsilon_{3}^{2}+c^{2} \epsilon_{1}^{2}\right)}}\right),
\end{aligned}
$$

where the material constants for this crystal are used in the second step. With this angle, the split vector $\vec{p}$ defined as the vector pointing from the ordinary to the extraordinary ray in the (10.4) plane for a crystal of thickness $t$ follows from

$$
\vec{p}=\frac{t}{\cos \alpha} \vec{S}_{\mathrm{e}}-t \vec{S}_{\mathrm{o}}
$$

This vector $\vec{p}$ is aligned along the [42. $\overline{1}$ ] direction as we find only one negative solution for

$$
\vec{p}=d \vec{e}_{b},
$$

namely,

$$
d=-\frac{2 \sqrt{3} a c t\left(\epsilon_{1}-\epsilon_{3}\right)}{12 a^{2} \epsilon_{3}+c^{2} \epsilon_{1}} \approx-0.11 t
$$

$\left(\epsilon_{1}>\epsilon_{3}\right.$ for calcite). This length is at the same time the split distance between the ordinary and the extraordinary ray in the (10.4) plane of calcite. The relation between the Poynting vectors, the calcite crystallographic directions and the split vector are visualized in the main text, Fig. 4(a). For a crystal of $t=5 \mathrm{~mm}$, we find a split length of about $0.55 \mathrm{~mm}$, which is visible by the bare eye.

\section{APPENDIX B: SHORT-RANGE VERTICAL FORCES}

The van der Waals force law given by Zanette et al. [37] was found to most reliably represent the long-range interaction in our data [24]. It describes the probe as a tip-side truncated pyramid of infinite height with a spherical cap terminating the truncated end. The opening angle of the pyramid measured to the center of each side plane is denoted as $\theta$ and the half-side length of the blunt pyramid end is $L$. The cap is a spherical segment with height $h_{1}$ of a virtually full sphere with radius $R$. We eliminated the fitting parameter $L$ by geometric relations between the quantities [24]. In the course of our analysis, we furthermore fixed the tip radius to $25 \mathrm{~nm}$, since this parameter is not independent from the other parameters. The shape of the resulting van der Waals force curve is, however, independent over a large regime of $R$; consequently we found no artifacts introduced by this approach. The tip-sample distance $z$ is measured from the lowest point of the spherical cap to the
TABLE III. Parameters resulting from the optimum fit of Eq. (B1) to the experimental mean curve $\overline{F_{z}}$.

\begin{tabular}{lcc}
\hline \hline cutoff point & $z_{\text {cut }}$ & $5.8 \AA$ \\
$z$ offset & $z_{\text {off }}$ & $0.13 \mathrm{~nm}$ \\
Hamaker constant & $\mathrm{H}$ & $0.61 \times 10^{-20} \mathrm{~J}$ \\
tip radius (fixed) & $\mathrm{R}$ & $25 \mathrm{~nm}$ \\
half-opening angle & $\theta$ & $77.2^{\circ}$ \\
spherical cap height & $h_{1}$ & $1.2 \mathrm{~nm}$ \\
half width of truncated end & $L$ & $7.56 \mathrm{~nm}$ \\
uncertainty force minimum position & $\delta z_{\min }$ & $0.4 \AA$ \\
uncertainty force minimum value & $\delta F_{\min }$ & $60 \mathrm{pN}$ \\
uncertainty zero force position & $\delta z_{\text {zero }}$ & $0.4 \AA$ \\
\hline \hline
\end{tabular}

sample surface represented by an infinite plane. We include a fit parameter $z_{\text {off }}$ to account for a shift along the $z$ axis with respect to the short-range interaction. The van der Waals force law is given by [24,37]

$$
\begin{aligned}
F_{\mathrm{vdW}}\left(z ; H, R, \theta, h_{1}, z_{\mathrm{off}}\right) \\
=-\frac{H}{6}\left[\frac{h_{1}^{2}\left(3 R \hat{z}+(R-\hat{z}) h_{1}\right)}{\hat{z}^{2}\left(\hat{z}+h_{1}\right)^{3}}+\frac{R^{2}-\left(R-h_{1}\right)^{2}}{\left(\hat{z}+h_{1}\right)^{3}}\right. \\
\left.\quad+\frac{4 \tan (\theta)\left(\sqrt{R^{2}-\left(R-h_{1}\right)^{2}}+\tan (\theta)\left(\hat{z}+h_{1}\right)\right)}{\pi\left(\hat{z}+h_{1}\right)^{2}}\right]
\end{aligned}
$$

with $\hat{z}=z+z_{\text {off }}$. We fit this force law to the experimental mean curve $\overline{F_{z}}$, which is calculated as the average curve along the horizontal $y$ direction:

$$
\overline{F_{z}}(z)=\frac{1}{M} \sum_{j=1}^{M} F_{z}\left(y_{j}, z\right) .
$$

The resulting fit parameters are listed in Table III.

\section{APPENDIX C: SUPPLEMENTARY DATA FOR THE 2D FORCE SLICE}

All measurement channels for the data presented in Figs. 5-7 are reproduced in Fig. 11. No site-specific contrast is apparent in the amplitude data shown in Fig. 11(c), instead we find pure white noise with $\sigma \approx 1.5 \mathrm{pm}$. With the amplitude constant to better than $0.01 \%$, we find that the Sader-Jarvis force recovery strategy given in Eq. (1) is fully justified. A small site-specific contrast is observed in the dissipation $\Gamma(z)$ data in Fig. 11(d). These data were converted to units of eV/cycle using Eq. (1) in Ref. [53]. In our dataset, the dissipative contribution raises to a maximum of around $180 \mathrm{meV} /$ cycle at very close tip-sample distances. We find the largest dissipation within this data set next to the identified point defect as indicated in Fig. 5 by a dashed yellow circle. It is noteworthy to mention that although we measure a dissipative interaction, no change in the amplitude is apparent. 

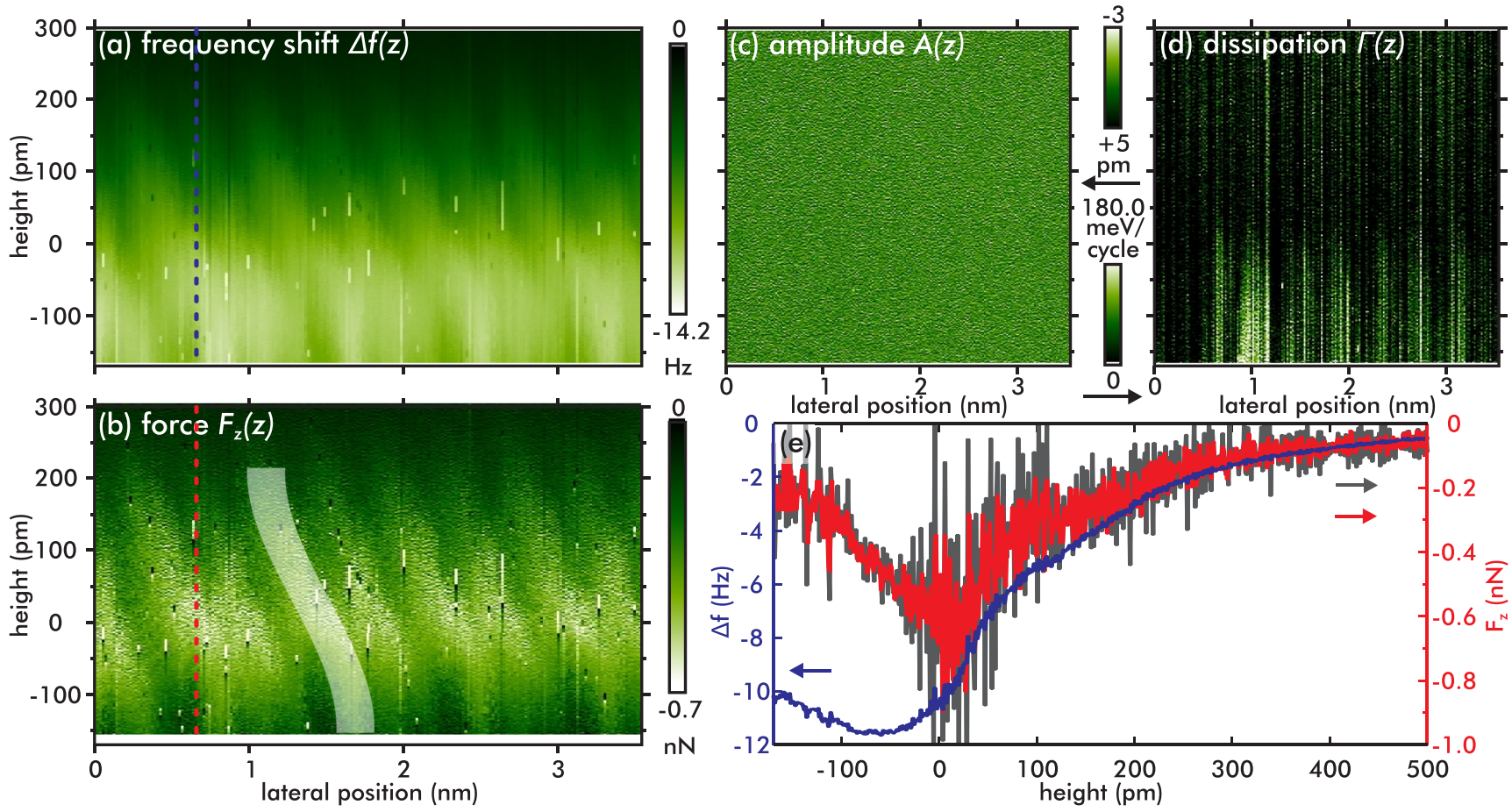

FIG. 11. (Color online) (a) Frequency shift $\Delta f$, (b) vertical total force interaction $F_{z}$, (c) amplitude $A$, and (d) dissipation $\Gamma$ data. The data are represented with a stretched vertical axis for better representation. (e) Frequency shift (dark blue), recovered force (gray) as well as Lanczos and Savitzky-Golay filtered force (red) data extracted from positions marked by dotted vertical lines in the respective slice data.

[1] G. Binnig, H. Rohrer, C. Gerber, and E. Weibel, Phys. Rev. Lett. 49, 57 (1982).

[2] G. Binnig, C. F. Quate, and Ch. Gerber, Phys. Rev. Lett. 56, 930 (1986).

[3] F. J. Giessibl, Science 267, 68 (1995).

[4] G. H. Simon, T. König, M. Nilius, H. P. Rust, M. Heyde, and H.-J. Freund, Phys. Rev. B 78, 113401 (2008).

[5] C. Barth and M. Reichling, Nature (London) 414, 54 (2001).

[6] P. Rahe, M. Kittelmann, J. L. Neff, M. Nimmrich, M. Reichling, P. Maass, and A. Kühnle, Adv. Mater. 25, 3948 (2013).

[7] J. V. Barth, G. Costantini, and K. Kern, Nature (London) 437, 671 (2005).

[8] L. Gross, F. Mohn, N. Moll, P. Liljeroth, and G. Meyer, Science 325, 1110 (2009).

[9] L. Gross, Nat. Chem. 3, 273 (2011).

[10] B. Such, T. Glatzel, S. Kawai, S. Koch, and E. Meyer, J. Vac. Sci. Technol. B 28, C4B1 (2010).

[11] S. Kawai, T. Glatzel, S. Koch, A. Baratoff, and E. Meyer, Phys. Rev. B 83, 035421 (2011).

[12] A. S. Foster, A. L. Shluger, and R. M. Nieminen, Appl. Surf. Sci. 188, 306 (2002).

[13] C. Barth, A. S. Foster, C. R. Henry, and A. L. Shluger, Adv. Mater. 23, 477 (2011).

[14] R. Bennewitz, S. Schär, V. Barwich, O. Pfeiffer, E. Meyer, F. Krok, B. Such, J. Kolodzej, and M. Szymonski, Surf. Sci. 474, L197 (2001).

[15] W. A. Deer, R. A. Howie, and J. Zussman, An Introduction to the Rock Forming Minerals (Pearson Education Limited, Harlow, England, 1992).
[16] P. Rahe, J. Schütte, and A. Kühnle, J.f Phys.: Condens. Matter 24, 084006 (2012).

[17] S. Kawai, C. M. Pina, A. Bubendorf, G. Fessler, T. Glatzel, E. Gnecco, and E. Meyer, Nanotechnol. 24, 055702 (2013).

[18] G. Meyer and N. M. Amer, Appl. Phys. Lett. 53, 1045 (1988).

[19] T. R. Albrecht, P. Grütter, D. Horne, and D. Rugar, J. Appl. Phys. 69, 668 (1991).

[20] Y. Sugimoto, T. Namikawa, K. Miki, M. Abe, and S. Morita, Phys. Rev. B 77, 195424 (2008).

[21] P. Rahe, J. Schütte, W. Schniederberend, M. Reichling, M. Abe, Y. Sugimoto, and A. Kühnle, Rev. Sci. Instrum. 82, 063704 (2011).

[22] M. Abe, Y. Sugimoto, T. Namikawa, K. Morita, N. Oyabu, and S. Morita, Appl. Phys. Lett. 90, 203103 (2007).

[23] J. E. Sader and S. P. Jarvis, Appl. Phys. Lett. 84, 1801 (2004).

[24] S. Kuhn and P. Rahe, Phys. Rev. B 89, 235417 (2014).

[25] B. J. Albers, T. C. Schwendemann, M. Z. Baykara, N. Pilet, M. Liebmann, E. I. Altman, and U. D. Schwarz, Nat. Nanotechnol. 4, 307 (2009).

[26] D. W. Pohl and R. Möller, Rev.f Sci.ic Instrum. 59, 840 (1988).

[27] M. Abe, Y. Sugimoto, O. Custance, and S. Morita, Nanotechnol. 16, 3029 (2005).

[28] U. Dürig, Appl. Phys. Lett. 75, 433 (1999).

[29] F. J. Giessibl, Appl. Phys. Lett. 78, 123 (2001).

[30] J. Welker, E. Illek, and F. J. Giessibl, Beilstein J. Nanotechnol. 3, 238 (2012). 
[31] J. Sader and S. Jarvis, Determination of force and energy vs seperation from frequency vs seperation data, http://www. nanofunction.org/storage/resources-images/techniques/fmafm/fmafm_Sader.nb (2004), downloaded 25.09.2011.

[32] G. H. Simon, M. Heyde, and H. P. Rust, Nanotechnol. 18, 255503 (2007).

[33] J. Lübbe, M. Temmen, P. Rahe, A. Kühnle, and M. Reichling, Beilstein J. Nanotechnol. 4, 227 (2013).

[34] A. Sweetman and A. Stannard, Beilstein J. Nanotechnol. 5, 386 (2014).

[35] M. A. Lantz, H. J. Hug, R. Hoffmann, P. J. A. van Schendel, P. Kappenberger, S. Martin, A. Baratoff, and H. J. Güntherodt, Science 291, 2580 (2001).

[36] M. Nonnenmacher, M. P. O’Boyle, and H. K. Wickramasinghe, Appl. Phys. Lett. 58, 2921 (1991).

[37] S. I. Zanette, A. O. Caride, V. B. Nunes, G. L. Klimchitskaya, F. L. Freire, and R. Prioli, Surf. Sci. 453, 75 (2000).

[38] Carbonates: Mineralogy and Chemistry, edited by R. J. Reeder, Reviews in Mineralogy Vol. 11 (Mineralogical Society of Amer, Chantilly, VA, 1983).

[39] H. Effenberger, K. Mereiter, and J. Zemann, Zeitschrift Für Kristallographie 156, 233 (1981).

[40] R. M. Hazen, T. R. Filley, and G. A. Goodfriend, Proc. Natl. Acad. Sci. USA 98, 5487 (2001).

[41] A. L. Rachlin, G. S. Henderson, and M. C. Goh, Am. Mineral. 77, 904 (1992).
[42] P. Rahe, S. Kuhn, and A. Kühnle, J. Unsolved Quest. 3, 21 (2013).

[43] S. L. Stipp and M. F. Hochella, Geochimica Et Cosmochimica Acta 55, 1723 (1991).

[44] J. Schütte, P. Rahe, L. Tröger, S. Rode, R. Bechstein, M. Reichling, and A. Kühnle, Langmuir 26, 8295 (2010).

[45] S. Fremy, S. Kawai, R. Pawlak, T. Glatzel, A. Baratoff, and E. Meyer, Nanotechnol. 23, 055401 (2012).

[46] S. Kawai, T. Glatzel, S. Koch, B. Such, A. Baratoff, and E. Meyer, Phys. Rev. B 81, 085420 (2010).

[47] A. S. Foster, A. Y. Gal, Y. J. Lee, A. L. Shluger, and R. M. Nieminen, Appl. Surf. Sci. 210, 146 (2003).

[48] Y. Liang, A. S. Lea, D. R. Baer, and M. H. Engelhard, Surf. Sci. 351, 172 (1996).

[49] G. Teobaldi, K. Lämmle, T. Trevethan, M. Watkins, A. Schwarz, R. Wiesendanger, and A. L. Shluger, Phys. Rev. Lett. 106, 216102 (2011).

[50] A. J. Weymouth, T. Hofmann, and F. Giessibl, Science 343, 1120 (2014).

[51] Handbook of Chemistry and Physics, edited by R. Weast and M. E. Astle, 62nd ed. (CRC Press, Bocy Raton, FL, 1981).

[52] B. Saleh and M. Teich, Fundamentals of Photonics, Wiley Series in Pure and Applied Optics (Wiley, New York, 2007).

[53] G. Langewisch, W. Kaminski, D. A. Braun, R. Möller, H. Fuchs, A. Schirmeisen, and R. Perez, Small 8, 602 (2012).

[54] P. Rahe, R. Bechstein, and A. Kühnle, J. Vac. Sci. Technol. B 28, C4E31 (2010). 